\title{
A Fractionation-based Local Activation Wave Detector for Atrial Electrograms of Atrial Fibrillation
}

\author{
Diego Osorio ${ }^{1}$, Raúl Alcaraz ${ }^{2}$, José J Rieta ${ }^{1}$ \\ ${ }^{1}$ BioMIT.org, Electronic Engineering Department, Universitat Politecnica de Valencia, Spain \\ ${ }^{2}$ Research Group in Electronic, Biomedical and Telecomm. Eng., Univ. of Castilla-La Mancha, Spain
}

\begin{abstract}
Atrial fibrillation (AF) electrograms (EGMs) present high heterogeneity of morphologies and amplitudes that turns the detection of their local activation waves (LAWs) a very hard task to perform. In this study, a novel fractionation-based LAW detector for bipolar EGMs is introduced. The method modifies traditional Botteron's band-pass filtering, decreasing its low cut-off frequency from 40 to $20 \mathrm{~Hz}$, thus benefiting slow local activations detection. Furthermore, high and low amplitude activations in complex fractionated atrial electrograms (CFAEs) are equalized, thus facilitating the detection of low amplitude activations. A minimum refractory period of $50 \mathrm{~ms}$ is imposed between activations, however, additional minor activations are later sought for intervals longer than the median cycle length. All the LAWs from a set of 40 real bipolar EGMs, mostly CFAEs, were manually annotated by expert physicians and served to evaluate performance. Detections closer than 40 ms to a manual annotation were considered as correct. Detection results provided $95.41 \%$, 92.13\% and $96.37 \%$ in Sensitivity, Accuracy and Precision for CFAEs, respectively, whereas for less fractionated EGMs they were $100 \%$ in any case. Therefore, the new $L A W$ detector has provided robust performance even under highly fractionated EGMs.
\end{abstract}

\section{Introduction}

Atrial fibrillation (AF) is the most common cardiac arrhythmia in clinical practice, which also represent the highest expense among all the cardiovascular diseases with a $15 \%$ of total costs [1]. Nowadays, AF is present in approximately $4 \%$ of the population aged $60-70$ years and $10-17 \%$ of those aged over 80 years [2]. Moreover, a total of 14 to 17 million patients are estimated to suffer from AF for 2030 in the European Union [3]. These evidences arise the need to develop further research on the mechanisms triggering and sustaining AF aimed at improving its unsatisfactory treatment [3].
Among the several therapies for the treatment of AF, catheter ablation (CA) is the first line therapy for the restoration of sinus rhythm. This technique relies on the application of scars in the endocardium by using highenergy radio-frequency signals to stop the ectopic electrical activity that sustains AF [4]. Electrograms (EGMs) are intracardiac recordings of the heart that can be employed to characterize atrial activity during $\mathrm{AF}$ guiding $\mathrm{CA}$ procedures through the use of modeling techniques able to locate possible ablation targets [5]. To this respect, the most common focus of ectopic electrical activity provoking AF are the pulmonary veins (PVs) [6], thus being priority targets for ablation. Although PVs ablation obtains effective results for paroxysmal $\mathrm{AF}$, the ablation of additional ectopic foci beyond the PVs in persistent AF patients may be necessary. For this purpose, one strategy relies on the search and ablation of complex fractionated atrial electrograms (CFAEs) [7]. CFAEs can identified by the tracing of atrial regions with atrial cycle length (CL) lower than 120 $\mathrm{ms}$. The CL is computed as the average distance between two consecutive atrial activations and serves to estimate the depolarization frequency of a certain region [8].

The proposed method is based on a hybrid strategy performing a first amplitude-based detection of most prominent local activations waves (LAW). Next, a detection of low amplitude LAWs within CLs wider than the median CL is carried out. Two main features have also been included. Firstly, a less aggressive preprocessing than traditional Botteron's [9] approach has been applied, thus facilitating the detection of slow LAWs. Secondly, the presence of CFAEs is sensed and their wide amplitude variability reduced before thresholding for LAW detection. These two steps, together with the detection algorithm, have achieved high performance in LAW detection under CFAEs. Additionally, since the algorithm is applied over 5-second overlapped sections of the EGM under processing, many of its settings, such as the detection thresholds, evolve in the same way as the local fractionation. Hence, the method may be applied over long EGMs, regardless of their duration or evolving fractionation. 

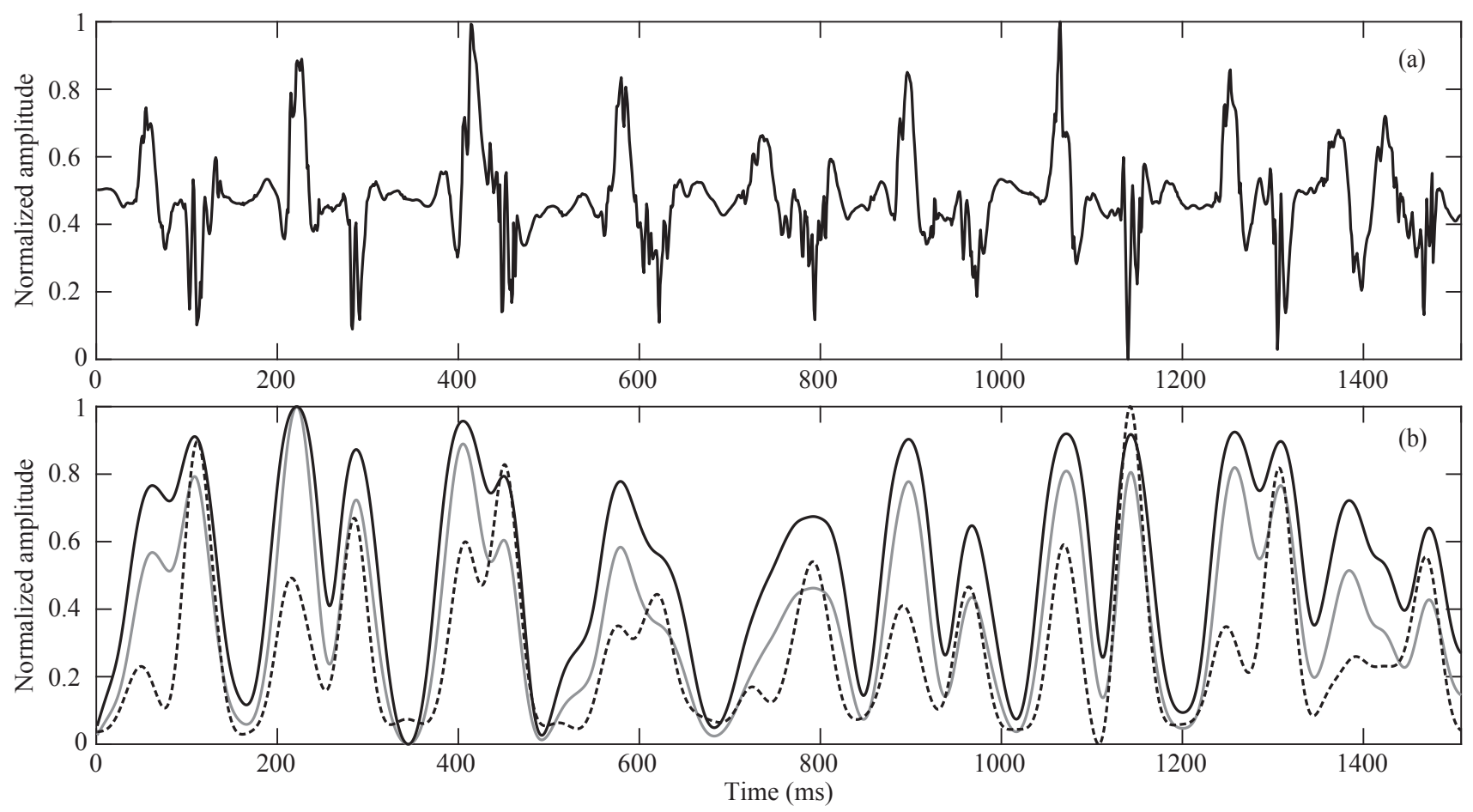

Figure 1. Comparison between Botteron's preprocessing and both the band-pass filter cut-off frequency modification and the amplitude equalization introduced by the proposed method. (a) Denoised CFAE (type III AF). (b) Resulting signals from the different preprocessings. The dashed line corresponds Botteron's approach, the continuous grey line is this method's outcomes before CFAE amplitude equalization and the black continuous line is the final output after CFAE equalization.

\section{Methods}

\subsection{Dataset and preprocessing}

A dataset of 40 EGMs was obtained employing a CardioLab system (General Electric, Wauwatosa, WI, USA) after written consent of patients undergoing CA procedures. EGMs were resampled at $1 \mathrm{kHz}$ and came filtered by an $50 \mathrm{~Hz}$ notch and a $0.5-500 \mathrm{~Hz}$ band-pass hardware filters embedded in the recording system. Two expert physicians manually annotated the dataset and classified the EGMs by AF types according to the Wells' classification [10], resulting in a set of 5 type I, 7 type II and 28 type III AF EGMs. Then, the EGMs were denoised through a Wavelet-based strategy, which performed better than regular filtering both in denoising and preserving EGM morphology [11].

The first stage of the proposed method is based on the preprocessing introduced in 1995 by Botteron and Smith [9]. This algorithm begins with the application of a $40-250 \mathrm{~Hz}$ band-pass filter, followed by a rectification of the resulting signal, and a final $20 \mathrm{~Hz}$ low-pass filtering. This technique creates proportional waveforms to the amplitude of the EGM components with frequencies within the interval of the band-pass filter cut-offs. However, the implementation proposed here replaces the low cut-off fre- quency of the band-pass filter from 40 to $20 \mathrm{~Hz}$. This reduction is made with the intention of achieving a better response for the slow components of some activations that would unavoidably result in very low amplitude LAWs, which are more difficult or even impossible to detect by an amplitude criterion. As an example, Figure 1 plots a CFAE together with the resulting signals after different preprocessing stages. Observe, specially in times 600 and $1400 \mathrm{~ms}$, how the proposed methodology will notably facilitate threshold detection of low amplitude LAWs.

\subsection{Specific processing of CFAEs}

After preprocessing, the next stage of the method is a novel kurtosis-based strategy for the classification of EGMs according to their degree of fractionation. Since CFAEs are the EGMs with larger amplitude variability, this will serve to apply a specific signal equalization to CFAEs aimed at facilitating the later detection stage. The equalization consists of an enhancement of low amplitude activations but, at the same time, maintaining the values of high amplitude activations, thus reducing the typical dynamic range on CFAEs. For small values, the enhancement is approximately double, while for the highest values the variation is not significant. The resulting signal of this pro- 


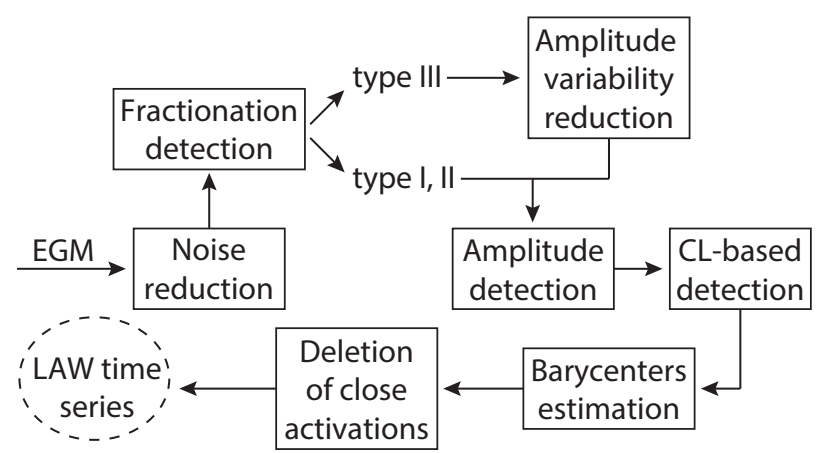

Figure 2. Flowchart of the stages composing the detection process of the method.

cess presents activations which are now easier to detect by amplitude thresholding. This evidence allows to set easy amplitude thresholds for the detection of most activations. The application of equalization to low fragmented EGMs (type I and II AF) is not recommended since their LAW detection is much easier. Moreover, experimental equalization tests over the entire dataset has shown that a few detection errors were introduced in some type II EGMs.

After proper equalization, a thresholding stage is applied to the EGM to get the most prominent activations and compute the median CL. This value will later serve as the reference for the second detection stage in which tiny or missed LAWs are sought under CLs longer than the average CL, as will be next detailed.

\subsection{Local activation waves detection}

First, EGMs are divided in 5-second length segments with a $25 \%$ of overlapping to endow the method with a detection adaptability to potential fractionation variations along the EGM. Thus, for each segment, the main LAWs detection is initiated by thresholding once the EGM has been preprocessed and, in case of CFAEs, properly equalized. Those activations with a maximum peak value higher than the threshold are added to the set of detected activations. If two activations are closer than $50 \mathrm{~ms}$, which is the established minimum refractory period between activations, the one with the highest amplitude is preserved and the lower one is deleted from the set.

Then, the median CL of the segment is estimated and employed as the reference condition for the next stage. This second step is based on a progressive analysis of those intervals with CLs longer than the median CL. The process is repeated until there are no more intervals to analyze. Since the longer the interval, the more likely that undetected activations lay within it, the amplitude threshold is decreased proportionally to the interval's duration.

Once the detection algorithm is ended, the method estimates the barycenter of each detected LAW with the aim
Table 1. Detection results of the LAW detector for the different types of electrograms. Results are provided as mean \pm standard deviation in percentage.

\begin{tabular}{cccc}
\hline $\begin{array}{c}\text { Type of } \\
\text { EGM }\end{array}$ & $\begin{array}{c}\text { Accuracy } \\
(\%)\end{array}$ & $\begin{array}{c}\text { Sensitivity } \\
(\%)\end{array}$ & $\begin{array}{c}\text { Precision } \\
(\%)\end{array}$ \\
\hline \hline Type I & $100.00 \pm 0.00$ & $100.00 \pm 0.00$ & $100.00 \pm 0.00$ \\
Type II & $100.00 \pm 0.00$ & $100.00 \pm 0.00$ & $100.00 \pm 0.00$ \\
Type III & $92.13 \pm 5.45$ & $95.41 \pm 4.67$ & $96.37 \pm 2.96$ \\
\hline
\end{tabular}

to set more precisely the activation times. The barycenters are computed as the mean value of the area of each rectified LAW, which are isolated in 70-ms windows for this computation step [12]. Moreover, the method employs this stage to check if two adjacent activations have tended to converge and the current distance between them is shorter than the $50 \mathrm{~ms}$ established, in which case the lowest activation is deleted from the activations set. The different stages of the detection process and the evolution of the algorithm are indicated in the flowchart of Figure 2.

\subsection{Performance evaluation}

The performance of this new LAW detector was evaluated by comparing the detected activations with the manual annotations made by physicians. Those activations closer than $40 \mathrm{~ms}$ to an annotation were considered as correct detections. Then, sensitivity, accuracy and precision were calculated to provide detailed results of the method's performance for type I, II and III EGMs.

\section{Results}

Detection results for the different EGM types are shown in Table 1. As can be seen, results for type I and type II EGMs were ideal, since the method did not commit mistakes during the detection process. Furthermore, the $92.13 \%$ in accuracy obtained for type III EGMs, together with just a $5.45 \%$ of standard deviation, really confers to this method an outstanding robustness in every possible scenario. Lastly, detection errors for type III EGMs were $4.59 \%$ for under-detected activations and $3.33 \%$ for overdetected activations, on average.

\section{Discussion}

The novel amplitude equalization applied to CFAEs employed by this method, together with the decrease of the low cut-off frequency of the band-pass filter from 40 to 
$20 \mathrm{~Hz}$, has entailed a clear facilitation of the detection stage. This equalization has served to employ a fixed amplitude threshold for the main amplitude detection with outstanding results. This improvement in signal preprocessing before thresholding detection has demonstrated to facilitate the detection of some slow, small activations typically present in CFAEs (see Fig. 1). In addition, detection results were higher than $92 \%$ in any case, thus suggesting this LAW detector as a precise CL estimator [13] with potentially could be applied for guiding CA procedures [7].

Furthermore, the hybrid strategy for the detection stage of a main amplitude detection followed by a detection based on the CLs' duration has showed a very robust performance. The highest activations are detected by amplitude and the lowest ones by their location in CLs longer than the median CL of the EGM. Finally, the segmentation of the EGMs, as well as the estimation of the fractionation degree, allow the method to be constantly adapting to the EGM characteristics. This adaptation process serves to deal properly with possible transition periods between AF types and to perform more precise detections, which ensures accurate results regardless of the EGMs length.

\section{Conclusions}

A novel LAW detector focused on bipolar EGMs has been introduced. The method has demonstrated to provide accurate detections even for very fragmented EGMs. Its adaptability to fragmentation variations, together with the precise results achieved, makes this method a good candidate to be used during real time applications in clinical practice as CL estimator assisting CA procedures.

\section{Acknowledgements}

Research supported by grants TEC2014-52250-R and DPI2017-83952-C3 MINECO/AEI/FEDER, UE.

\section{References}

[1] Wodchis WP, Bhatia RS, Leblanc K, Meshkat N, Morra D. A review of the cost of atrial fibrillation. Value in Health 2012;15(2):240 - 248

[2] Zoni-Berisso M, Lercari F, Carazza T, Domenicucci S. Epidemiology of atrial fibrillation: European perspective. Clinical Epidemiology 2014;6(1):213-220.

[3] Kirchhof P, Benussi S, Kotecha D, Ahlsson A, et al. 2016 ESC guidelines for the management of atrial fibrillation developed in collaboration with EACTS. European Heart Journal 2016;37(38).

[4] Gerstenfeld EP, Duggirala S. Atrial fibrillation ablation: Indications, emerging techniques, and follow-up. Progress in Cardiovascular Diseases 2015;58(2):202-212.

[5] Baumert M, Sanders P, Ganesan A. Quantitativeelectrogram-based methods for guiding catheter ablation in atrial fibrillation. Proceedings of the IEEE 2016; 104(2):416-431.

[6] Bhatt HV, Fischer GW. Atrial fibrillation: Pathophysiology and therapeutic options. Journal of Cardiothoracic and Vascular Anesthesia 2015;29(5):1333-1340.

[7] Kumagai K, Sakamoto T, Nakamura K, et al. Combined dominant frequency and complex fractionated atrial electrogram ablation after circumferential pulmonary vein isolation of atrial fibrillation. Journal of Cardiovascular Electrophysiology 2013;24(9):975-983.

[8] Bollmann A, Husser D, Mainardi L, Lombardi F, Langley P, Murray A, Rieta JJ, Millet J, Olsson SB, Stridh M, Sörnmo L. Analysis of surface electrocardiograms in atrial fibrillation: Techniques, research, and clinical applications. Europace 2006;8(11):911-926.

[9] Botteron GW, Smith JM. A technique for measurement of the extent of spatial organization of atrial activation during atrial fibrillation in the intact human heart. IEEE Transactions on Biomedical Engineering 1995;42(6):579-586.

[10] Wells Jr JL, Karp RB, Kouchoukos NT, MacLean WAH, James TN, Waldo AL. Characterization of atrial fibrillation in man: Studies following open heart surgery. Pacing and Clinical Electrophysiology 1978;1(4):426-438.

[11] Martínez-Iniesta M, Ródenas J, Alcaraz R, Rieta JJ. Waveform integrity in atrial fibrillation: The forgotten issue of cardiac electrophysiology. Annals of Biomedical Engineering 2017;45(8):1890-1907.

[12] Faes L, Nollo G, Antolini R, Gaita F, Ravelli F. A method for quantifying atrial fibrillation organization based on wave-morphology similarity. IEEE Transactions on Biomedical Engineering 2002;49(12 I):1504-1513.

[13] Osorio D, Alcaraz R, Rieta JJ. Comparative study of methods for atrial fibrillation cycle length estimation in fractionated electrograms. In Computing in Cardiology Conference (CinC), volume 44. IEEE, 2017; In press.

Address for correspondence:

Diego Osorio

Electronic Engineering Department, Building 7F

Universidad Politécnica de Valencia

Camino de Vera, s/n, 46022, Valencia, Spain

E-mail: dosorio@upv.es 